\title{
CONTRASTE ENTRE EL PERFIL DEL POSTULANTE Y LOS ALUMNOS DE PRIMER AÑO EN 2005. UNIVERSIDAD CATÓLICA DE LA SANTÍSIMA CONCEPCIÓN
}

\section{Introducción}

Dadas las particularidades del sistema nacional de admisión, al cual está adscrito la Universidad Católica de la Santísima Concepción, la definición de un perfil de ingreso no ha tenido gran relevancia, ya que la selección y matrícula depende de los resultados de la Prueba de Selección Universitaria (PSU). En la publicidad de la institución, sin embargo, se explicita un "Perfil del Postulante"l, para informar a los futuros estudiantes. Además, en las distintas ponderaciones exigidas para las pruebas de selección se encuentra implícita una idea del perfil que los estudiantes deben cumplir.

A partir de esas informaciones y las disponibles en las bases de datos de la Dirección de Admisión y Registro Académico, se realizaron tres análisis: a) de los conceptos y características generales expresadas en la publicidad respecto del perfil del postulante, b) de la coherencia entre lo declarado en el perfil del postulante y las ponderaciones exigidas, y c) del grado de adecuación entre ambos aspectos y las características de los estudiantes matriculados.

En este sentido, aun cuando este artículo es un estudio de caso y su aporte para generalizar es limitado, explora una dimensión metodológica hasta ahora escasamente tratada en nuestra realidad: la posibilidad de operacionalizar un perfil de ingreso para los estudiantes.

1 En este artículo se considera los términos "perfil de ingreso" y "perfil del postulante" como sinónimos. 


\section{El perfil como concepto y requisito general}

La palabra "perfil" es definida por la Real Academia Española como el "conjunto de rasgos peculiares que caracterizan a una persona o cosa". En la educación superior su sentido es un poco más amplio y se le entiende como el conjunto de características referidas a conocimientos, habilidades, valores y actitudes que se demandan de un estudiante para acceder a una carrera determinada. Estos atributos están fuertemente ligados a la naturaleza de la(s) disciplina(s) base de una profesión en particular. Su empleo radica en la necesidad de conocer, de antemano, los requerimientos básicos que demanda el estudio de una carrera, con el fin de servir de orientación en el proceso de postulación y como referente del de formación.

En general, en los procesos de selección universitaria existe una declaración formal de los rasgos que se espera reúnan los estudiantes para acceder a una carrera en particular, pero, en la práctica, esto se suele definir operacionalmente en las ponderaciones que se asignan a los puntajes de las distintas pruebas que conforman el sistema de admisión a las universidades chilenas (PSU).

Este es también el caso de la Universidad Católica de la Santísima Concepción: no existen perfiles de ingreso definidos en los planes de estudios de las carreras, sino que se realiza una especificación en la publicidad y una operacionalización en las ponderaciones exigidas a las distintas pruebas de la PSU.

\section{El perfil de ingreso como requerimiento de las universidades del Consejo de Rectores}

La definición de perfiles de ingreso, en relación con las exigencias particulares de la formación en una determinada carrera, sólo tiene sentido en la medida en que la selección de estudiantes para esa especialidad opera en función de esa definición y si el proceso formativo la considera sustantivamente. 
La Comisión Nacional de Acreditación (CNAP) no señala explícitamente el requerimiento de un perfil de ingreso. Sin embargo, la demanda de las instituciones, al señalar que se requiere una coherencia entre el proceso de selección y los posteriores, bajo el criterio de efectividad del proceso enseñanza-aprendizaje: "La unidad debe poseer criterios de admisión claramente establecidos, públicos y apropiados a las exigencias de su plan de estudios. El proceso de enseñanza debe tomar en cuenta las competencias de los estudiantes y los requerimientos del plan de estudios, proporcionando oportunidades de aprendizaje teóricas y prácticas, según corresponda (...)

La unidad debe establecer claramente sus criterios y mecanismos de admisión de alumnos. Estos deben ser de conocimiento público y apropiados para que los estudiantes tengan oportunidad de alcanzar el grado y título profesional en un plazo razonable.

La unidad debe realizar un diagnóstico de la preparación de los alumnos que ingresan a la carrera, tomando en consideración los resultados de dicho diagnóstico en el diseño del plan de estudios y en el establecimiento de instancias de nivelación iniciales y oportunas" 2 .

Un factor determinante de la escasa relevancia que ha tenido este tema en Chile, es el rol que tiene la PSU (antes la Prueba de Aptitud Académica, PAA) como elemento definitorio de la selección de alumnos.

El diseño del sistema de selección de alumnos del Consejo de Rectores posee ciertas características que no favorecen la existencia de perfiles de ingreso reales y operativos, es decir, que no sean retóricos ni con fines publicitarios. Estas características, someramente, son las siguientes:

a) Existe un sistema nacional de selección y matrícula que opera bajo normas consensuadas entre las instituciones y que regula la

2 CNAP Criterios Genéricos de Evaluación de Carreras Profesionales. Disponible en www.cnap.cl 
competencia entre éstas. Vale decir, el sistema no sólo organiza y administra la aplicación del instrumento y la distribución y certificación de resultados, sino que continúa como sistema conjunto en las etapas de postulación, selección y matrícula.

b) Las personas no pueden elegir directamente la universidad y la carrera, sino que realizan una secuencia de postulaciones, que sirve como insumo para que las instituciones las seleccionen en una de ellas.

c) La selección opera casi exclusivamente por el puntaje ponderado obtenido del rendimiento en la PSU. Los postulantes se ordenan según ese puntaje y, de acuerdo a cupos previamente establecidos por las universidades, pueden ser seleccionados o no en una opción.

Lo más relevante de este diseño, en relación con el perfil, son dos aspectos. En primer lugar y en estricto rigor, los alumnos no eligen la universidad y la carrera, sino que la selección opera desde la perspectiva e intereses de las instituciones, favoreciendo a las que son más prestigiosas y, entre ellas, a las carreras de mayor demanda.

En segundo lugar, el sistema prescinde de todas aquellas dimensiones y cualidades de una persona que no son medidas por la PSU, salvo en el caso de la aplicación de pruebas especiales previas $^{3}$. La coordinación e integración del sistema hasta el proceso de postulación y matrícula exige la homogeneización de los criterios con que opera la selección en las 25 instituciones.

En suma, después de la etapa de postulación, el estudiante espera ver "si queda" y las universidades esperan ver "cómo les fue". Entretanto, un algoritmo computacional realiza la selección de alumnos, de acuerdo con parámetros prefijados, basados en los puntajes de la PSU.

3 La aplicación de pruebas especiales ha disminuido en el tiempo. En 1996 existían 11 universidades que las aplicaban en 34 carreras; en 2005 sólo seis en 11 carreras. 
Como puede verse, el sistema se funda, tácitamente, en que la aplicación de una prueba nacional (ya sea alineada con el currículo de enseñanza media o centrada en la evaluación de aptitudes) es necesaria y suficiente para resolver con justicia y pertinencia la selección de alumnos para las carreras universitarias, eligiendo a los más capaces para enfrentar con éxito los estudios superiores.

Dicho fundamento, sin embargo, sólo tiene validez si:

a) La oferta es significativamente menor que la demanda, lo que obliga a seleccionar a los mejores egresados de la enseñanza media.

b) La calidad del proceso de educación media cumple estándares mínimos de eficacia y lo hace con razonable homogeneidad, de manera que se pueda afirmar que la gran mayoría de los que egresan de ella, si no todos, está en condiciones de enfrentar estudios superiores. Esto justifica elegir a los "mejores" de un grupo donde todos son "elegibles".

c) La PSU tiene validez para medir los conocimientos, habilidades y actitudes que demanda el currículo de la enseñanza media, y es suficientemente versátil para detectar diferentes aspectos de las personas que interesan en los perfiles de egreso.

La primera premisa se cumple sólo en determinadas carreras e instituciones selectivas. Se está llegando a una relación de un postulante por cada vacante ofrecida. De ese modo, ya existen carreras, en las universidades del Consejo de Rectores, en las que la selección mediante puntajes de la PSU tiene un carácter meramente formal. Algo similar ocurre en la mayoría de las universidades privadas.

La segunda premisa tampoco se cumple. No todos los egresados de la enseñanza media reciben una formación que cumpla estándares mínimos de calidad, con lo cual se puede afirmar que la verdadera selección ocurre en la enseñanza preterciaria. Por otra parte, el descenso de la selectividad de las carreras obliga a que la docencia universitaria deba hacerse cargo de estudiantes con 
grandes necesidades de nivelación académica y cultural. Para tal cometido, cada vez más se requiere conocer otras facetas del perfil de estos estudiantes como insumo para la adecuada organización de la docencia.

La tercera premisa tampoco se cumple, puesto que no se definen perfiles de ingreso y, aún definiéndose, la PSU no está diseñada para medir todos los aspectos cognitivos y actitudinales ni para entregar información fina. Su reporte es un puntaje estandarizado que sólo permite ordenamientos. Adicionalmente, las instituciones suelen validar esta modalidad de selección de alumnos mediante estudios del nivel de predictividad del rendimiento universitario a través de la PSU, tratando de identificar los mejores predictores y la mejor combinación de ponderaciones entre ellos. De esta forma, la PSU no opera como un elemento de aproximación a un perfil de ingreso, sino a la inversa: sólo desde la óptica predictiva.

El diseño descrito pierde relevancia en la medida en que la educación superior es cada vez menos selectiva en su conjunto, manteniendo esta característica sólo en carreras e instituciones donde la demanda supera ampliamente a la oferta. En los casos en que éstas se igualan o la demanda es menor, se podría perfectamente prescindir de este sistema o complementarse con otros elementos que concurran a la toma de decisiones.

En este escenario, las instituciones en general no han elaborado perfiles de ingreso más allá de las dimensiones formales asociadas a la publicidad, ya que la selección se delega en el sistema nacional integrado de postulación, selección y matrícula.

\section{La metodología}

La Universidad Católica de la Santísima Concepción no define perfiles de ingreso para sus estudiantes en relación con los contenidos de los planes de estudios, sino sólo con el fin de orientar 
a los postulantes, usando para ello la publicidad que elabora la Dirección de Extensión y Comunicaciones.

Esta definición, sin embargo, no se realiza para la totalidad de las carreras. Por esta razón, en el análisis se trabajó con 11 de las 21 que ofrecieron vacantes en el año 2005, contenidas en la tabla 1:

Tabla 1

Carreras analizadas

\begin{tabular}{|l|l|}
\hline Facultad o escuela & Carrera \\
\hline Escuela de Periodismo & Periodismo \\
\hline Facultad de Ciencias & Biología Marina \\
\hline Facultad de Ciencias de la Salud & $\begin{array}{l}\text { Medicina } \\
\text { Enfermería } \\
\text { Nutrición y Dietética }\end{array}$ \\
\hline Facultad de Ciencias Económicas y Administrativas & Ingeniería Comercial \\
\hline Facultad de Derecho & Derecho \\
\hline Facultad de Educación & Trabajo Social \\
\hline & $\begin{array}{l}\text { Ingeniería Civil } \\
\text { Facultad de Ingeniería }\end{array}$ \\
& Ingeniería Civil Informática \\
\hline
\end{tabular}

El análisis se realizó en tres niveles. En primer lugar, se examinaron las principales categorías utilizadas por las distintas carreras en la definición de su perfil del postulante. Enseguida, se evaluó la forma como éstas operacionalizan dicho perfil dentro de las posibilidades que ofrece la batería de pruebas de la PSU, es decir, se analizó el grado de coherencia entre lo declarado en la publicidad y las ponderaciones que se otorgan a los conocimientos y competencias exigidos. Finalmente, se contrastaron ambas dimensiones con las características de los estudiantes matriculados en ellas. En este caso, se consideró el puntaje obtenido en la PSU y, como parámetro de medición, la media nacional situada en 500 puntos. 


\section{El perfil de ingreso contenido en la publicidad y su operacionalización en la PSU}

Los perfiles de ingreso, definidos por las carreras para orientar a los postulantes, hacen referencia a aspectos cognitivos relacionados con la(s) disciplina(s) base de la carrera y a algunos aspectos actitudinales y valóricos asociados al ejercicio de la profesión ${ }^{4}$.

En la tabla 2 se aprecian en detalle estos perfiles y las dimensiones que incluyen. Para construir éstas se utilizaron los siguientes criterios: a) se caracterizó como cognitiva cuando hace referencia a una ciencia específica o a algún ámbito genérico que pueda ser aproximado a una ciencia, b) como actitudinal cuando lo que prima es algún tipo de motivación, y c) como valórica cuando a la actitud o motivación subyace una referencia directa a determinados valores.

Tabla 2

Dimensiones contenidas en el perfil del postulante

\begin{tabular}{|l|l|l|l|}
\hline Facultad & Carrera & Perfil del postulante & Dimensión \\
\hline $\begin{array}{l}\text { Escuela de } \\
\text { Periodismo }\end{array}$ & Periodismo & $\begin{array}{l}\text { 1. Interés por conocer la información } \\
\text { contingente } \\
\text { 2. manifestar capacidad de observación de } \\
\text { la realidad inmediata y de la relación } \\
\text { de los distintos hechos sociales } \\
\text { 3. habilidad para comunicarse en forma } \\
\text { oral y escrita }\end{array}$ & 2. Cognitiva \\
\hline $\begin{array}{l}\text { Facultad de } \\
\text { Ciencias }\end{array}$ & $\begin{array}{l}\text { Biología } \\
\text { Marina }\end{array}$ & $\begin{array}{l}\text { 1. Persona motivada por el conocimiento } \\
\text { de la naturaleza } \\
\text { 2. interesada en promover su cuidado } \\
\text { 3. en aprovechar su productividad }\end{array}$ & $\begin{array}{l}\text { 1. Cognitiva } \\
\text { 2. Valórica } \\
\text { 3. Valórica }\end{array}$ \\
\hline
\end{tabular}

4 Sólo la carrera de Enfermería incluye una referencia a un estado de salud compatible. 


\begin{tabular}{|c|c|c|c|}
\hline \multirow[t]{3}{*}{$\begin{array}{l}\text { Facultad de } \\
\text { Ciencias de } \\
\text { la Salud }\end{array}$} & Medicina & $\begin{array}{l}\text { 1. Interés por las ciencias biológicas } \\
\text { 2. manifestar una profunda vocación de } \\
\text { servicio } \\
\text { 3. con un respeto intransable por la vida }\end{array}$ & $\begin{array}{l}\text { 1. Cognitiva } \\
\text { 2. Valórica } \\
\text { 3. Valórica }\end{array}$ \\
\hline & Enfermería & $\begin{array}{l}\text { 1. Interés por las ciencias biológicas } \\
\text { 2. humanísticas y sociales } \\
\text { 3. profunda vocación de servicio para } \\
\text { atender a las personas en estado de } \\
\text { salud y enfermedad } \\
\text { 4. espíritu de superación } \\
\text { 5. entusiasmo } \\
\text { 6. salud compatible }\end{array}$ & $\begin{array}{l}\text { 1. Cognitiva } \\
\text { 2. Cognitiva } \\
\text { 3. Valórica } \\
\text { 4. Actitudinal } \\
\text { 5. Actitudinal } \\
\text { 6. Física }\end{array}$ \\
\hline & $\begin{array}{l}\text { Nutrición } \\
\text { y Dietética }\end{array}$ & $\begin{array}{l}\text { 1. Interés por el estudio del área biológica } \\
\text { 2. espíritu de superación } \\
\text { 3. capacidad comunicacional } \\
\text { 4. profunda vocación de servicio } \\
\text { 5. respeto por la salud de las personas }\end{array}$ & $\begin{array}{l}\text { 1. Cognitiva } \\
\text { 2. Actitudinal } \\
\text { 3. Cognitiva } \\
\text { 4. Valórica } \\
\text { 5. Valórica }\end{array}$ \\
\hline $\begin{array}{l}\text { Facultad } \\
\text { de Ciencias } \\
\text { Económicas y } \\
\text { Administrativas }\end{array}$ & $\begin{array}{l}\text { Ingeniería } \\
\text { Comercial }\end{array}$ & $\begin{array}{l}\text { 1. Condiciones de líder } \\
\text { 2. habilidades matemáticas } \\
\text { 3. humanas } \\
\text { 4. iniciativa } \\
\text { 5. características de emprendedor }\end{array}$ & $\begin{array}{l}\text { 1. Actitudinal } \\
\text { 2. Cognitiva } \\
\text { 3. Valórica } \\
\text { 4. Actitudinal } \\
\text { 5. Actitudinal }\end{array}$ \\
\hline $\begin{array}{l}\text { Facultad de } \\
\text { Derecho }\end{array}$ & Derecho & $\begin{array}{l}\text { 1. Vocación de servicio a la sociedad } \\
\text { 2. inspirado en un permanente afán de } \\
\text { superación } \\
\text { 3. búsqueda de la verdad a través de la } \\
\text { justicia y la cultura }\end{array}$ & $\begin{array}{l}\text { 1. Valórica } \\
\text { 2. Actitudinal } \\
\text { 3. Valórica }\end{array}$ \\
\hline $\begin{array}{l}\text { Facultad de } \\
\text { Educación }\end{array}$ & $\begin{array}{l}\text { Trabajo } \\
\text { Social }\end{array}$ & $\begin{array}{l}\text { 1. Actitud de compromiso frente a la } \\
\text { problemática social } \\
\text { 2. capacidad para establecer relaciones } \\
\text { humanas positivas expresadas con } \\
\text { respeto } \\
\text { 3. tolerancia y aceptación de los otros } \\
\text { 4. habilidad para integrar equipos } \\
\text { 5. asumir roles organizativos y } \\
\text { administrativos } \\
\text { 6. ejercer liderazgo en su ámbito de } \\
\text { trabajo } \\
\text { 7. vocación de servicio a la sociedad } \\
\text { 8. inspirado en un permanente afán de } \\
\text { superación } \\
\text { 9. búsqueda de la verdad a través de la } \\
\text { justicia y la cultura }\end{array}$ & $\begin{array}{l}\text { 1. Valórica } \\
\text { 2. Valórica } \\
\text { 3. Valórica } \\
\text { 4. Actitudinal } \\
\text { 5. Actitudinal } \\
\text { 6. Actitudinal } \\
\text { 7. Valórica } \\
\text { 8. Actitudinal } \\
\text { 9. Valórica }\end{array}$ \\
\hline
\end{tabular}




\begin{tabular}{|c|c|c|c|}
\hline \multirow[t]{3}{*}{$\begin{array}{l}\text { Facultad de } \\
\text { Ingeniería }\end{array}$} & $\begin{array}{l}\text { Ingeniería } \\
\text { Civil }\end{array}$ & $\begin{array}{l}\text { 1. Habilidades matemáticas } \\
\text { 2. creatividad } \\
\text { 3. capacidad de abstracción } \\
\text { 4. perseverancia } \\
\text { 5. disciplina } \\
\text { 6. responsabilidad }\end{array}$ & $\begin{array}{l}\text { 1. Cognitiva } \\
\text { 2. Cognitiva } \\
\text { 3. Cognitiva } \\
\text { 4. Actitudinal } \\
\text { 5. Actitudinal } \\
\text { 6. Actitudinal }\end{array}$ \\
\hline & $\begin{array}{l}\text { Ingeniería } \\
\text { Civil } \\
\text { Informática }\end{array}$ & $\begin{array}{l}\text { 1. Habilidades matemáticas } \\
\text { 2. creatividad } \\
\text { 3. perseverancia } \\
\text { 4. responsabilidad } \\
\text { 5. disciplina }\end{array}$ & $\begin{array}{l}\text { 1. Cognitiva } \\
\text { 2. Cognitiva } \\
\text { 3. Actitudinal } \\
\text { 4. Actitudinal } \\
\text { 5. Actitudinal }\end{array}$ \\
\hline & $\begin{array}{l}\text { Ingeniería } \\
\text { Civil } \\
\text { Industrial }\end{array}$ & $\begin{array}{l}\text { 1. Habilidades matemáticas } \\
\text { 2. creatividad } \\
\text { 3. perseverancia } \\
\text { 4. responsabilidad } \\
\text { 5. disciplina }\end{array}$ & $\begin{array}{l}\text { 1. Cognitiva } \\
\text { 2. Cognitiva } \\
\text { 3. Actitudinal } \\
\text { 4. Actitudinal } \\
\text { 5. Actitudinal }\end{array}$ \\
\hline
\end{tabular}

Como es posible apreciar en la tabla 2, las carreras tienden a combinar de distinta manera dimensiones cognitivas, actitudinales y valóricas en lo que denominan el perfil del postulante. Esto permite hacer la siguiente clasificación:

Tabla 3

Dimensiones exigidas por carrera

\begin{tabular}{|l|l|}
\hline Dimensión & Carreras \\
\hline Cognitiva & Periodismo \\
\hline Cognitiva y actitudinal & $\begin{array}{l}\text { Ingeniería Civil } \\
\text { Ingeniería Civil Informática } \\
\text { Ingeniería Civil de Industrias }\end{array}$ \\
\hline Cognitiva y valórica & $\begin{array}{l}\text { Biología Marina } \\
\text { Medicina }\end{array}$ \\
\hline Cognitiva, actitudinal y valórica & $\begin{array}{l}\text { Enfermería } \\
\text { Nutrición y Dietética } \\
\text { Ingeniería Comercial }\end{array}$ \\
\hline Actitudinal y valórica & $\begin{array}{l}\text { Derecho } \\
\text { Trabajo Social }\end{array}$ \\
\hline
\end{tabular}

De acuerdo con la tabla 3, dos carreras (Derecho y Trabajo Social) deben ser excluidas del análisis que sigue, ya que en 
el perfil del postulante no especifican características de tipo cognitivo, susceptibles de ser operacionalizadas a través de los puntajes de la PSU.

En las tablas 4, 5 y 6 se muestra la relación entre la carrera, la dimensión científica a que hace referencia la dimensión cognitiva y las ponderaciones exigidas en la PSU, ordenadas de acuerdo con el grado de congruencia entre el perfil del postulante explicitado y las ponderaciones exigidas, distinguiendo tres categorías:

a) Carreras que presentan un alto grado de congruencia entre el perfil del postulante explicitado y las ponderaciones exigidas:

Tabla 4

\begin{tabular}{|l|l|c|c|c|c|c|}
\hline Carrera & Ámbito & Notas EM & Lenguaje & Matemáticas & Historia & Ciencias \\
\hline Periodismo & $\begin{array}{l}\text { Sociología/Ciencias } \\
\text { de la Información }\end{array}$ & 30 & 30 & 20 & 20 & \\
\hline Ingeniería Comercial & Matemáticas & 30 & 20 & 40 & 10 & \\
\hline Ingeniería Civil & Matemáticas & 30 & 20 & 40 & & 10 \\
\hline $\begin{array}{l}\text { Ingeniería Civil } \\
\text { Informática }\end{array}$ & Matemáticas & 30 & 20 & 40 & & 10 \\
\hline $\begin{array}{l}\text { Ingeniería Civil } \\
\text { Industrial }\end{array}$ & Matemáticas & 30 & 20 & 40 & & 10 \\
\hline
\end{tabular}

b) Carreras que presentan una congruencia intermedia entre el perfil del postulante explicitado y las ponderaciones exigidas:

Tabla 5

\begin{tabular}{|l|l|c|c|c|c|c|}
\hline Carrera & Ámbito & Notas EM & Lenguaje & Matemáticas & Historia & Ciencias \\
\hline Medicina & Ciencias Biológicas & 30 & 20 & 30 & & 20 \\
\hline Enfermería & $\begin{array}{l}\text { Ciencias Biológicas, } \\
\begin{array}{l}\text { Humanidades, } \\
\text { Ciencias Sociales }\end{array}\end{array}$ & 30 & 20 & 30 & & 20 \\
\hline $\begin{array}{l}\text { Nutrición y } \\
\text { Dietética }\end{array}$ & $\begin{array}{l}\text { Ciencias Biológicas, } \\
\text { Comunicación }\end{array}$ & 30 & 20 & 30 & & 20 \\
\hline
\end{tabular}


c) Carreras que presentan un grado significativo de incongruencia entre el perfil del postulante explicitado y las ponderaciones exigidas:

Tabla 6

\begin{tabular}{|l|l|c|c|c|c|c|}
\hline Carrera & Ámbito & Notas EM & Lenguaje & Matemáticas & Historia & Ciencias \\
\hline Biología Marina & Ciencias Naturales & 30 & 30 & 30 & & 10 \\
\hline
\end{tabular}

\section{El perfil de ingreso real en las dimensiones cognitivas definidas como relevantes}

A continuación se analiza la distribución de puntajes de los estudiantes ingresados en 2005, en aquellas pruebas que se aproximan a las definiciones cognitivas consideradas relevantes en el perfil del postulante. El parámetro de evaluación utilizado es el porcentaje de la distribución que se sitúa por encima de la media nacional de puntajes, que corresponde a 500 puntos $^{5}$; vale decir, se considera que existe un grado suficiente de coherencia con el perfil de ingreso en su dimensión cognitiva si el postulante seleccionado tiene un puntaje igual o superior a la media de la distribución nacional de los puntajes, en aquella PSU que se relaciona más con las características definidas en el perfil de ingreso.

Con el objeto de dar mayor claridad al análisis, los resultados de agruparon por prueba y no por carrera. Los gráficos utilizados son box-plots (cajas y pestañas) y muestran la distribución en cuarteles. La "caja" central va del 25 al 75\% de la distribución. Las "pestañas" representan el 25\% inferior y superior, respectivamente.

5 A partir del proceso de admisión de 2005, la PSU entrega un puntaje normalizado, con un mínimo de 150 puntos, un máximo de 850, una media de 500 y una desviación estándar de 110. Más información en: Honorable Consejo de Rectores de las Universidades Chilenas. Comité Técnico Asesor. Resultados de la Aplicación de la Prueba de Selección Universitaria. Admisión 2005. 
a) Prueba de Ciencias: como se pudo constatar, las carreras donde la PSU de Ciencias tiene relevancia en el perfil de ingreso son las de Medicina, Enfermería, Nutrición y Dietética y Biología Marina.

Gráfico 1

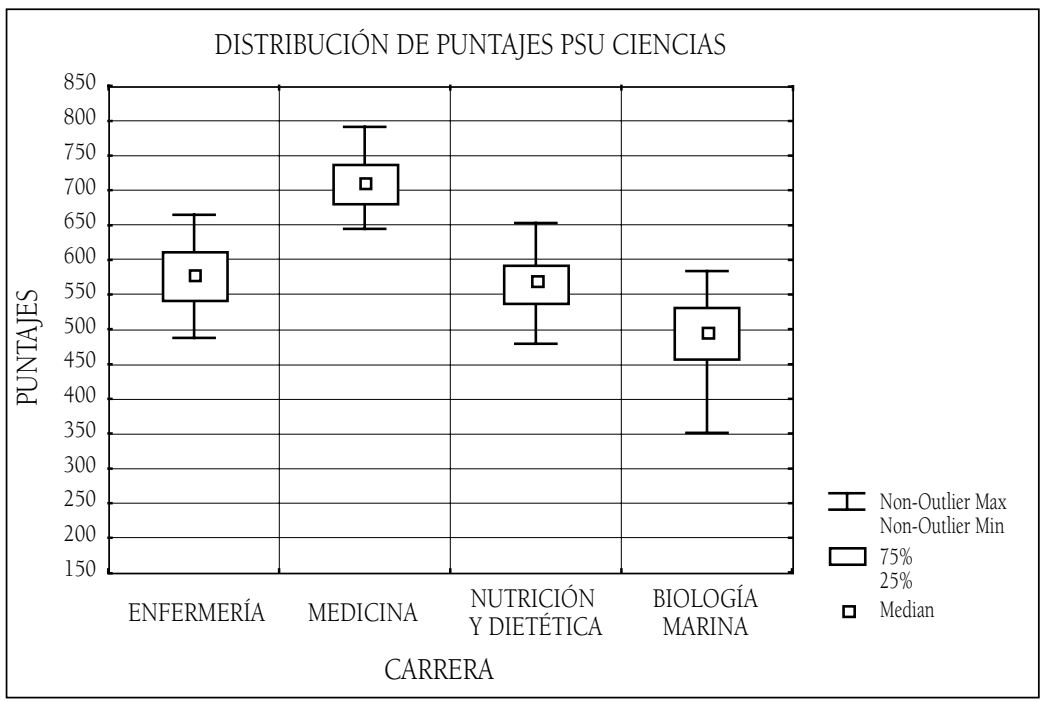

Como se aprecia en el gráfico 1, en la carrera de Medicina la totalidad de los alumnos ingresados en 2005 se encuentra por encima de la media nacional en esta prueba. Es más, se sitúan aproximadamente en el 10\% superior de los puntajes.

En Enfermería sólo un margen menor, correspondiente a cinco estudiantes de los ingresados en 2005, está bajo la media nacional de esta prueba, situándose ésta entre 550 y 600 puntos.

También, en Nutrición y Dietética la gran mayoría de los ingresados en 2005 se sitúa por encima del puntaje de referencia en esta prueba.

Por último, en Biología Marina la mitad de los estudiantes ingresados en 2005 no logra superar la media nacional en esta 
prueba, aunque la mediana de distribución está en los 500 puntos.

b) Prueba de Lenguaje y Comunicación: explicitada como un referente relevante en dos carreras: Nutrición y Dietética y Periodismo.

Gráfico 2

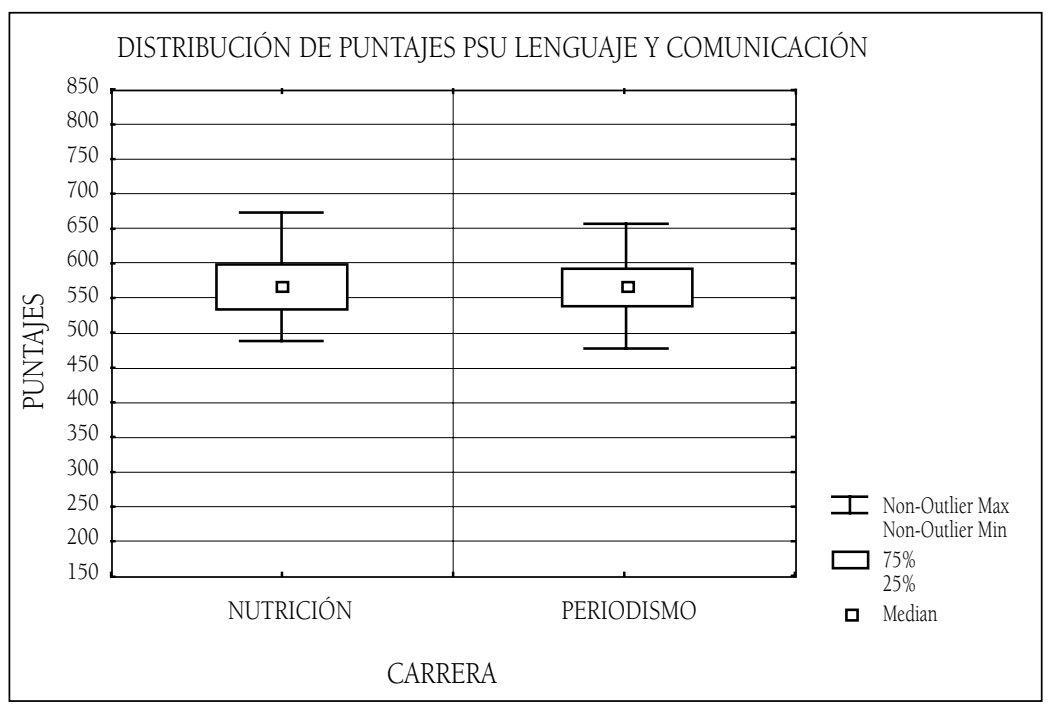

Como se aprecia en el gráfico 2, en Nutrición prácticamente la totalidad de los alumnos supera la media nacional.

En Periodismo existe también un comportamiento que guarda congruencia con el perfil de ingreso definido, ya que la mayor parte de los estudiantes se sitúan por encima del parámetro de la media nacional. Sin embargo, se observa el ingreso de alumnos que posiblemente requieran algún apoyo especial para superar la brecha entre sus capacidades y el perfil de ingreso. 
c) Prueba de Matemáticas: descrita como un referente relevante en cuatro carreras: tres de la Facultad de Ingeniería y una de la Facultad de Ciencias Económicas y Administrativas.

Gráfico 3

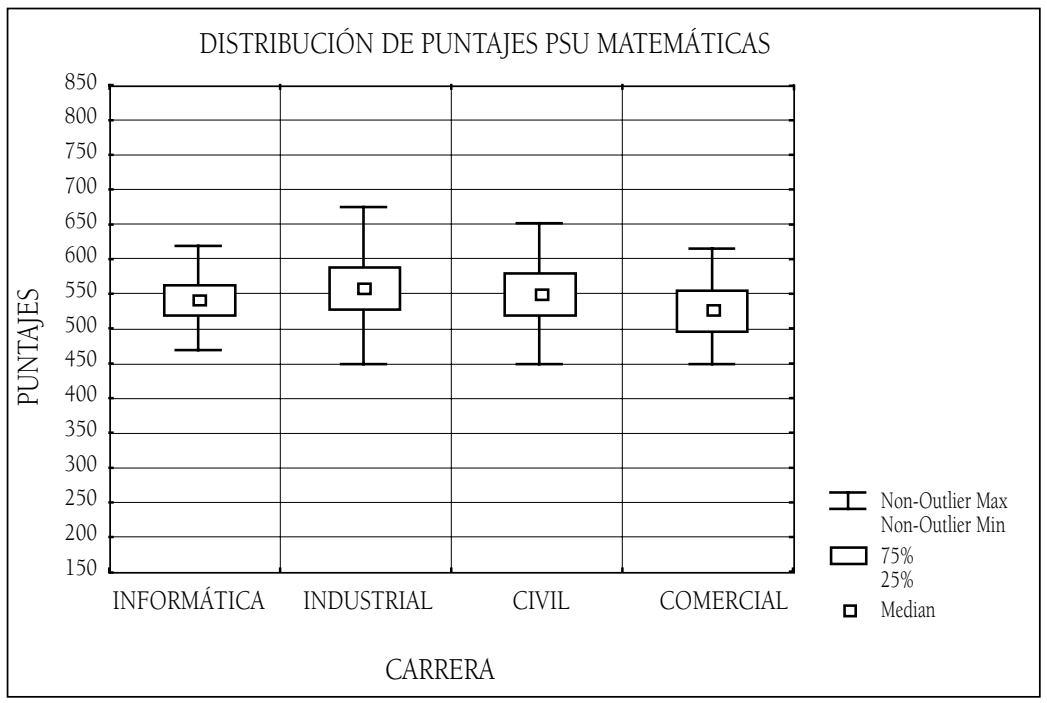

Como puede observarse en el gráfico 3, las carreras de Ingeniería, incluida Ingeniería Comercial, tienen entre un 13 y un $25 \%$ de los estudiantes ingresados bajo el parámetro de evaluación de los 500 puntos. Claramente existe una brecha significativa entre los resultados de la selección de alumnos mediante la PSU y el perfil del postulante explicitado. Las medianas de la distribución en estas carreras están bajo los 550 puntos, salvo en el caso de Ingeniería Civil Industrial que supera esa cifra levemente. 
d) Prueba de Historia y Ciencias Sociales: expresada como un referente relevante en la carrera de Periodismo.

Gráfico 4

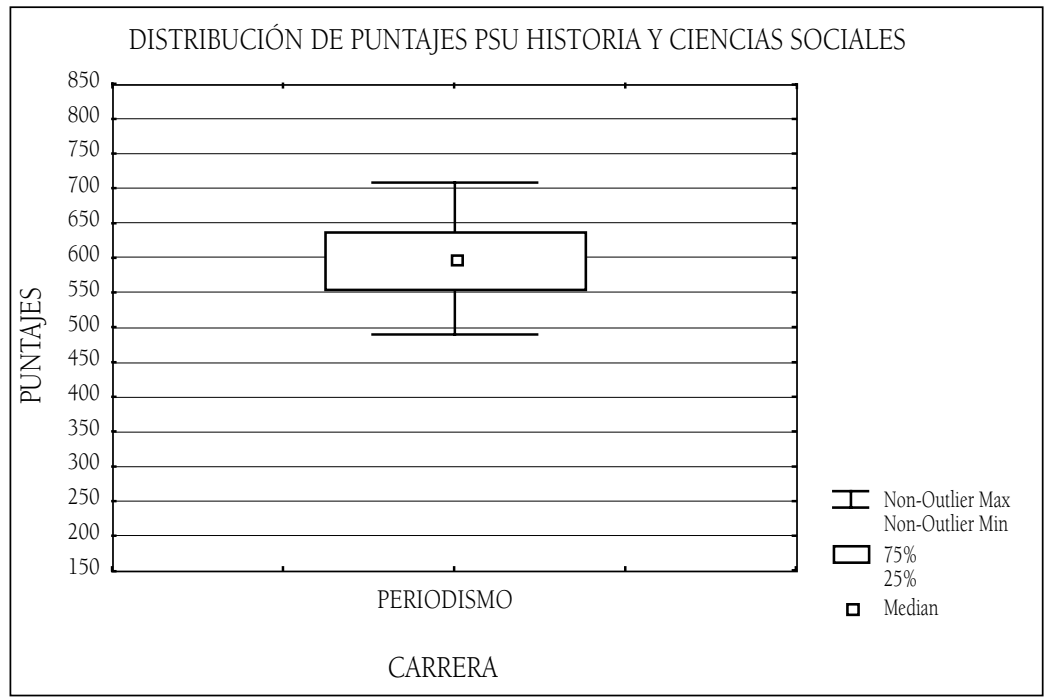

Se observa una alta congruencia, ya que muy pocos estudiantes están por debajo de los 500 puntos en el instrumento.

\section{Conclusiones}

Diversos factores asociados al proceso de postulación, selección y matrícula de los estudiantes de primer año a las universidades del Consejo de Rectores han determinado que el perfil de ingreso no sea considerado por las instituciones como un elemento relevante. Este sistema tiene un diseño operacional tal que, con independencia del tipo de pruebas que se aplique, no favorece procesos de selección referenciados por perfiles de ingreso, en el supuesto de que éstos existieran ${ }^{6}$. Incluso, las características estandarizadoras que impone

6 Es notable que en el debate que precedió al cambio de la PAA por la PSU, no haya tenido cabida el tema de la arquitectura básica del sistema, sin variaciones desde hace más de 30 años. 
un sistema conjunto que alcanza hasta las etapas de postulación, selección y matrícula se han visto aumentadas a partir del fin de la Prueba de Aptitud Académica (PAA) y su cambio por la PSU. En efecto, la primera tenía un esquema de instrumentos que, mediante una variedad de pruebas específicas optativas, tenía un mayor potencial de precisión en la selección por criterios cognitivos.

Por esta razón, las definiciones tienen un carácter formal y están asociadas más a la publicidad de las instituciones que a consideraciones reales que resulten inteligibles para una operacionalización efectiva. En conjunto, la definición de ponderaciones y la difusión para efectos de publicidad configuran, para algunas carreras, un perfil de ingreso más o menos explícito.

En la relación entre las definiciones asociadas a la publicidad y la operacionalización en la ponderación de puntajes de la PSU se presentan ciertas incongruencias, ya que, en el caso de la universidad en estudio, existen carreras que prescinden de la dimensión cognitiva, lo cual las hace ininteligibles desde las operaciones concretas de selección de alumnos.

Al analizar el perfil del postulante de las nueve carreras seleccionadas, es posible constatar que existe una significativa heterogeneidad entre dicha descripción y el esquema de las ponderaciones que definen para seleccionar a los nuevos alumnos. Ésta se da en dos sentidos: por una parte, las carreras enfatizan de manera distinta las dimensiones cognitivas, actitudinales y valóricas y, por otra, aun definiendo dimensiones cognitivas las ponderan de manera diversa.

En los resultados del proceso de selección, y considerando como hipótesis que existe coherencia con el perfil de ingreso en su dimensión cognitiva si el postulante seleccionado tiene un puntaje igual o superior a la media de la distribución nacional de los puntajes en aquella PSU que se relaciona más con las características 
definidas, se puede concluir que también en este ámbito existe heterogeneidad entre las carreras.

Si bien en algunas no se presenta una distancia significativa entre la dimensión cognitiva declarada como relevante en el perfil de ingreso (Medicina, Enfermería, Nutrición y Dietética y Periodismo), para la mayoría de éstas se puede afirmar que existen brechas entre el alumno real y el perfil de ingreso ideal de la carrera.

Una agenda de investigación y debate académico sobre este tema apunta hacia la pertinencia de los supuestos y operaciones en los cuales se resuelve la selección de alumnos a la educación superior, más que al debate sobre los instrumentos. El enfoque predictivo basado sólo en éstos tiene el sesgo de no poner bajo examen los procesos de enseñanza. La relevancia que suele tener el énfasis en la capacidad predictiva de los instrumentos quizás deba dar paso a procesos de selección que, sin dejar de ser prospectivos, incorporen de una manera más integral las características y perfil de ingreso del estudiante, para predecir el futuro, produciéndolo.

\section{Referencias bibliográficas}

Acosta, M., Bartolucci, R., Rodríguez, R. Perfil del alumno de primer ingreso al colegio de ciencias y humanidades. Universidad Autónoma de México. México 1981.

Beyer, Harald. Reflexiones preliminares sobre la prueba de selección a la universidad. En: Puntos de Referencia, 274, Centro de Estudios Públicos. Chile, enero de 2004.

CNAP Criterios genéricos de evaluación de carreras profesionales. Disponible en www.cnap.cl

CNAP Sistema Nacional de Aseguramiento de la Calidad de la Educación Superior. Santiago de Chile, 2002.

Donoso, Sebastián y Hawes, Gustavo. El sistema de selección de alumnos de las universidades chilenas: discusión de sus fundamentos, resultados y perspectivas. En: Education Policy Analysis Archives, 8(21), mayo 1, 2000,. Disponible en, http://epaa.asu.edu/epaa/v8n21.html 
Donoso, Sebastián y Hawes, Gustavo. 25 Años. La prueba. ¿Un proceso de selección?, CPU, Santiago de Chile, 1994.

Garduño, León. Hacia un modelo de evaluación de la calidad de instituciones de educación superior. En: Revista Iberoamericana de Educación, 21, Ediciones OEI.

Honorable Consejo de Rectores de las Universidades Chilenas. Comité Técnico Asesor. Resultados de la aplicación de la Prueba de Selección Universitaria. Admisión 2005. Santiago, 2005.

Schmitt, N. y Robert, I. Personnel Selection. En: Annual Review of Psychology, 41, 1990. 